Pacific Journal of Mathematics

BOUNDED MONOIDS

William Raymond Rico 


\title{
BOUNDED MONOIDS
}

\author{
WiLliam R. NiCO
}

\begin{abstract}
Monoids whose left $S$-sets $X$ always satisfy $\operatorname{sl}(X) \leqq h(X)$ are characterized in terms of chain conditions on principal left ideals.
\end{abstract}

For $S$ a monoid, a left $S$-set ( $S$-operand, $S$-system) is a set $X$ on which $S$ operates from the left and such that $1 x=x$ for all $x \in X$ where $1 \in S$ is the identity. For any $x \in X$, an $S$-subset of $X$ of the form $S x$ is called an orbit of $X$. It is well-known that every left $S$-set is a union of orbits and that, up to isomorphism, orbits are characterized by left congruences on $S$ (see [1], Chapt. 11).

In order to study the way orbits fit together in an $S$-set $X$ the author has in [2] and [3] constructed two chains of $S$-subsets (to be defined more fully below) of an $S$-set $X$, each having the property that the subquotients are essentially 0 -disjoint unions of orbits. The lengths of those two chains are denoted by $h(X)$ and $\operatorname{sl}(X)$. In [3] it is shown that when $h(X)$ is finite, then $\operatorname{sl}(X) \leqq h(X)$.

Let us call a monoid bounded if for every $S$-set $X$, one has $s l(X) \leqq h(X)$. Then a main goal of this paper is to show that a monoid is bounded if and only if there is a positive integer $n$ such that the monoid contains no proper chain of principal left ideals of length exceeding $n$.

1. Preliminaries, Let $X$ be a left $S$-set. An $S$-subset $Y$ of $X$ is a (possibly empty) subset $Y$ of $X$ such that $s y \in Y$ for all $s \in S$ and $y \in Y$. If $X$ and $Y$ are both orbits, we may say that $Y$ is a suborbit of $X$. If $Z$ is an $S$-set, a homomorphism $\phi: X \rightarrow Z$ is a function such that $\phi(s x)=s \phi(x)$ for all $x \in X$ and $s \in S$. A congruence $\sim$ on an $S$-set $X$ is an equivalence relation such that $x \sim y$ implies $s x \sim s y$ for $x, y \in X$ and $s \in S$. Denoting the set of congruence classes by $X / \sim$ one finds that $X / \sim$ is a left $S$-set under the induced action and is a homomorphic image of $X$ under the natural map $X \rightarrow X / \sim$.

If $Y \subset X$ is an $S$-subset, define a congruence $\sim_{Y}$ on $X$ by setting $x \sim_{Y} x^{\prime}$ if and only if $x=x^{\prime}$ or $x, x^{\prime} \in Y$. Let us denote $X / \sim_{Y}$ simply by $X / Y$. If $Y \neq \varnothing$, the class of $Y$ in $X / Y$ is denoted by 0 .

If $X$ is a left $S$-set, call $x, y \in X$ separated in $X$ if there is no $z \in X$ such that $x, y \in S z$. Then let us define a descending chain of $S$-subsets of $X$ by setting $X_{0}=X, X_{i+1}=\cup\left\{S x \cap S y: x, y \in X_{i}\right.$ are separated in $\left.X_{i}\right\}$ for $i>0$, and $X_{\sigma}=\bigcap\left\{X_{\tau}: \tau<\sigma\right\}$ for $\sigma$ a limit ordinal. Then $\operatorname{sl}(X)$, the saturation length of $X$, is the first ordinal 
$\alpha$ such that $X_{\alpha}=X_{\alpha+1}$. In general it need not be that $X_{\alpha}=\varnothing$, but whenever $S$ satisfies the ascending chain condition on orbits, it must be that $X_{\alpha}=\varnothing$ (see [2] and [3]).

Alternately, if one has an $S$-subset $Y \subset X$, then an element $x \in X$ is called $Y$-distinguished if for every $z \in X$ either $x \in S z$ or $z \in S x$ or $S x \cap S z \subset Y$. The orbit $S x \subset X$ is called $Y$-distinguished if $x^{\prime}$ is $Y$-distinguished for every $x^{\prime} \in S x$. Then one can define an ascending chain of $S$-subsets of $X$ by letting $X^{0}=\varnothing, X^{i+1}=\cup\{S x$ : $S x$ is an $X^{i}$-distinguished orbit of $\left.X\right\} \cup X^{i}$ for $i>0$, and $X^{o}=$ $\cup\left\{X^{\tau}: \tau<\sigma\right\}$ if $\sigma$ is a limit ordinal. Let $h(X)$, the height of $X$, be the first ordinal $\beta$ such that $X^{\beta}=X^{\beta+1}$. (Note: The definition of $Y$-distinguished orbit here is more restrictive than that used in [3] and corrects the definition given there in the sense that some (correctable) gaps in proofs these are trivially closed by the altered definition, the current definition being what the author had in mind in [3]. The principal difference is that in [3] $x^{1}=x$, whenever $x$ is an orbit, while here that need no longer be so. Here $h(X)$ is at least as in [3].) In general $X^{\beta} \neq X$, but if $S$ satisfies the descending chain condition on principal left ideals, then one always has $X^{\beta}=X$ (see [3]).

Throughout the rest of this paper we shall assume that $S$ satisfies the descending chain condition on principal left ideals. This assumption is justified by the following proposition.

Proposition 1.1. If $S$ does not satisfy the descending chain condition on principal left ideals, then there is a left $S$-set $X$ such that $h(X)=1$, but such that $\operatorname{sl}(X)=2$.

Proof. Suppose there is an infinite proper descending chain of principal left ideals in $S$. Denote it by $S \supsetneq S a_{1} \supsetneq S a_{2} \supsetneq \cdots$, and set $a_{0}=-1$. Let $I=\left\{x \in S\right.$ : for all $\left.i, a_{i} \notin S x\right\}$. Then if $I \neq \varnothing, I$ is a left ideal of $S$ which is maximal with respect to the property that for all $i=1,2, \cdots$ one has $a_{i} \notin I$. Form the left $S$-set $S \times N$, where $N=\{0,1, \cdots\}$, by letting $s(t, n)=(s t, n)$ for all $s, t \in S$ and $n \in N$.

Now let $X=(S \times N) / \sim$ where $\sim$ is the congruence on $S \times N$ given by $(s, m) \sim(t, n)$ if and only if either (i) $s, t \in I$ or (ii) $s=t \in$ $S a_{k}$ for some $k$ and $m, n \leqq k$. (In (ii) one wants to use the largest possible $k$.)

Let $[y, n] \in X$ denote the class of $(y, n) \in S \times N$. If $z \in I$, then since $[z, m]=[z, n]=\left[z^{\prime}, n\right]$ for all $m, n \in N$ and all $z^{\prime} \in I$ and since $s[z, m]=[s z, m]=[z, m]$, we can denote the class $[z, m]$ simply by 0 . (If $I=\varnothing$, all comments about 0 below are vacuous.) The $S$-set $X$ can be visualized schematically as in Figure 1 , in which the action by $S$ moves things downward along the indicated edges. 


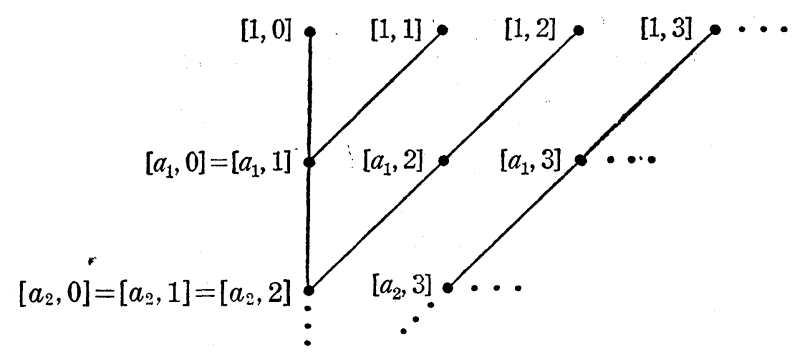

FIGURE 1

If $0 \neq[y, n] \in X$, then there is some $m$ such that $y \in S a_{m}$ but $y \notin S a_{m+1} \cup I$. By construction of $I$, there must be some $q$ such that $a_{q} \in S y$. Without loss of generality we may assume that $q>m$ and $q>n$. If $[y, n]=[y, q]$, we must have $n=q$ or $n, q \leqq m$, both of which are impossible by choice of $q$; hence $[y, n] \neq[y, q]$. However, since $n<q$, we find that $0 \neq\left[a_{q}, q\right]=\left[a_{q}, n\right] \in S[y, n] \cap S[y, q]$. Hence for every nonzero orbit $S[y, n]$ of $X$, there is an orbit incomparable to it (under inclusion) with which it has nonzero intersection. This says $X^{1}=\{0\}$ (or $X^{1}=\varnothing$ if $I=\varnothing$ ) and that no $[y, n] \neq 0$ is $X^{1}$ distinguished. Hence $X^{2}=X^{1}$, and $h(X) \leqq 1$.

On the other hand one easily sees that the elements $[1, n] \in X$ are all distinct for $n \in N$ and that these are the generators of the maximal orbits of $X$. Moreover, it is easy to see that for $n \neq m$ one has $S[1, n] \cap S[1, m] \subset S\left[a_{1}, 0\right] \cup\{0\}$. While $\left[a_{1}, 0\right] \in S[1,0] \cap S[1,1]$. Hence $X_{1}=S\left[a_{1}, 0\right] \cup\{0\}$, which is either an orbit or the disjoint union of two orbits (depending on whether or not $0 \in S\left[a_{1}, 0\right]$ ). Thus $X_{2}=\varnothing$, which gives $\operatorname{sl}(X)=2$.

Hence we will assume in what follows that $S$ satisfies the descending chain condition on principal left ideals.

For any monoid $S$ we now define two functions from $S$ to $N \cup\{\infty\}$. Let $h=h_{S}: S \rightarrow N \cup\{\infty\}$ be given by $h(x)=\sup \{n$ : there exists $a_{1}, a_{2}, \cdots, a_{n} \in S$ such that $\left.S x=S a_{1} \supsetneq S a_{2} \supsetneq \cdots \supsetneq S a_{n}\right\}$ for $x \in S$, and let $d=d_{S}: S \rightarrow N \cup\{\infty\}$ be given by $d(x)=\sup \{n$ : there exist $a_{1}, a_{2}, \cdots, a_{n} \in S$ such that $\left.S x=S a_{1} \varsubsetneqq S a_{2} \varsubsetneqq \cdots \varsubsetneqq S a_{n}\right\}$ for $x \in S$. We can call $h(x)$ the height of $x$ in $S$ and $d(x)$ the depth of $x$ in $S$.

2. Bounded monoids. We wish to characterize monoids $S$ such that $\operatorname{sl}(X) \leqq h(X)$.for every $S$-set $X$. . The following theorem justifies calling such monoids bounded, and we may take any one of its equivalent conditions as a definition of bounded monoid.

THEOREM 2.1. For a monoid $S$ the following are equivalent. (i) $\operatorname{sl}(X) \leqq h(X)$ for every S-set $X$. 
(ii) There exists an integer $n$ such that $S$ contains no proper chain of principal left ideals of length exceeding $n$.

(iii) $S$ satisfies the descending chain condition on principal left ideals and $h(X)<\infty$ for every $S$-set $X$.

(iv) $h(a)<\infty$ for every $a \in S$.

( $v) S$ satisfies the descending chain condition on principal left ideals and $d_{T}(\alpha)<\infty$ for every $a \in T=S / I$ where $I=\{x \in S: S x$ is a minimal left ideal\}.

Proof. One sees immediately that (ii) implies (iv) and (ii) implies (v).

That (iii) implies (i) follows from Theorem 5 of [3] provided one observes that the descending chain condition on principal left ideals of $S$ implies that every left $S$-set satisfies the descending chain condition on orbits (the hypothesis used in [3]). This follows since any chain $S x_{1} \supset S x_{2} \supset \cdots$ of orbits in an $S$-set $X$ gives rise to a chain of principal left ideals $S=S a_{1} \supset S a_{2} \supset \cdots$ in $S$ where $a_{1}=1$ and $a_{i+1} \in S a_{1}$ is such that $a_{i+1} x_{1}=x_{i+1}$ for $i \geqq 1$. Thus if $\phi: S \rightarrow X$ is the homomorphism of $S$-sets given by $\phi(s)=s x_{1}$ for $s \in S$, then $\phi\left(a_{i}\right)=x_{i}$ for all $i=1,2, \cdots$. Since the chain of left ideals $S a_{1} \supset$ $S a_{2} \supset \cdots$ must terminate, so must the chain of orbits $S x_{1} \supset S x_{2} \supset \cdots$.

To see that (iv) implies (ii) one need only observe that since $S=S 1$ is a principal left ideal containing every other principal left ideal, one can have no proper chain of principal left ideals of length exceeding $h(1)$.

To show that (v) implies (iv) it suffices to show that $h(1)<\infty$. Now $I=\{x \in S: S x$ is a minimal left ideal in $S\}$ is a two-sided ideal in $S$. In $T=S / I$, denote the image of $x \in S$ by $\bar{x} \in T$. Let $a_{1}=1$, $a_{2}, \cdots, a_{n} \in S$ be such that $S a_{1} \supsetneq S a_{2} \supsetneq \cdots \supsetneq S a_{n}$. Since $S$ satisfies the descending chain condition on principal left ideals we may assume that $S a_{n}$ is minimal, i.e., that $a_{n} \in I$. Then in $T$ one has $T \bar{a}_{1} \supsetneq T \bar{a}_{2} \supsetneq \cdots ? T \bar{a}_{n-1} \supsetneq\{0\}$. This says that $n \leqq d_{T}(0)$. Hence, in turn one can conclude that $h(1)=\sup \left\{n: S=S a_{1} \supsetneq \cdots \supsetneq S a_{n}\right\} \leqq$ $d_{T}(0)<\infty$, which establishes (iv).

To show that (iv) implies (iii) we observe that since (iv) implies (ii), $S$ must satisfy the descending chain condition on principal left ideals. We now show that for every $S$-set $X$ one has $\mathrm{h}(X) \leqq h(1)=n$. To do this let $X$ be an $S$-set and for $x \in X$, let $h_{X}(x)=\sup \{n: S x=$ $S x_{1} \supsetneq S x_{2} \supsetneq \cdots ? S x_{n}$ for $\left.x_{1}, \cdots, x_{n} \in X\right\}$. If $\psi: S \rightarrow S x$ is the homomorphism of $S$-sets given by $\psi(s)=s x$ for $s \in S$ and $x \in X$, and if $S x=S x_{1} \supsetneq S x_{2} \supsetneq \cdots ? S x_{m}$ is a proper descending chain of suborbits of $S x$, then there are $a_{1}, \cdots, a_{m} \in S$ such that $\psi\left(a_{i}\right)=x_{i}$ for $i=1, \cdots, m$ and such that $S=S a_{1} \supseteq S a_{2} \supseteq \cdots ? S a_{m}$. Hence, since we may take $a_{1}=1$, this shows that $m \leqq h(1)=n$. Therefore for 
every $x \in X$, one finds that $h_{X}(x) \leqq n$. Let $X(k)=\left\{x \in X: h_{X}(x) \leqq k\right\}$ for $k=1, \cdots, n$. Hence $X(1) \subset X(2) \subset \cdots \subset X(n)=X$.

To complete the proof that $h(X) \leqq n$, we now show by induction that $X(k) \subset X^{k}$ for $k=1, \cdots, n$. We observe first that $h_{X}(s x) \leqq h_{X}(x)$ for all $x \in X$ and all $s \in S$. Hence $X(k)$ is an $S$-subset of $X$ for all $k=1, \cdots, n$. Since $X(1)$ must be the union of the minimal orbits of $X$, one finds that $X(1) \subset X^{1}$. Now if $X(k) \subset X^{k}$ and if $x \in X(k+1)$ with $x \notin X^{k}$, then $s x$ must be an $X(k)$-distinguished orbit (for every proper suborbit of $S x$ must lie in $X(k))$. Hence $S x$ must be an $X^{k}$-distinguished orbit, which shows that $x \in X^{k+1}$, and hence that $X(k+1) \subset X^{k+1}$ as desired. But thus $X^{n} \supset X(n)=X$, which says that $k(X) \leqq n$.

Finally we show that (i) implies (ii). By virtue of Proposition 1.1, we know that $S$ must satisfy the descending chain condition on principal left ideals. We now complete the proof that (i) implies (ii) in two steps. First we show that if there is a proper chain of principal left ideals of $S$ of length $n$, then there is a left $S$-set $V_{n}$ such that $\operatorname{sl}\left(V_{n}\right)=h\left(V_{n}\right)=n$. Second we show that if such $V_{n}$ can be constructed for all $n \geqq 1$, then we can construct a left $S$-set $X$ such that $h(X)=\omega$ while $\operatorname{sl}(X)=\omega+1$, where $\omega$ in the first infinite ordinal. Since this contradicts (i), it must be that there is a positive integer $n$ such that $S$ contains no proper chain of principal left ideals of length exceeding $n$, which is the statement of (ii). We now proceed with the construction.

Suppose that $S a_{1}^{\prime} \subsetneq S a_{2}^{\prime} \varsubsetneqq S a_{3}^{\prime} \varsubsetneqq \cdots \varsubsetneqq S a_{n}^{\prime}$ is a proper chain of principal left ideals. Let $U_{1}^{\prime}=\{x \in S$ : $S x$ is a minimal left ideal $\}$, and for $1 \leqq i \leqq n-1$ let $U_{i+1}^{\prime}=U_{i}^{\prime} \cup\{x \in S$ : $S x$ is minimal such that $\left.x \notin U_{i}^{\prime}\right\}$. If $U_{\imath}^{\prime} \neq S$, then $U_{i}^{\prime} \varsubsetneqq U_{i+1}^{\prime}$ since $S$ satisfies the descending chain condition on principal left ideals. However, we observe that $a_{2}^{\prime} \notin U_{1}^{\prime}$, and inductively, that if $\alpha_{k}^{\prime} \notin U_{k-1}^{\prime}$, then $a_{k+1}^{\prime} \notin U_{k}^{\prime}$ for $2 \leqq k \leqq$ $n-1$. Hence we have a proper chain of left ideals $U_{1}^{\prime} \subsetneq \cdots \sqsubseteq U_{n}^{\prime}$. Observe now that if $x \in U_{k}^{\prime}$ and $x \notin U_{k-1}^{\prime}$ for $2 \leqq k \leqq n$, then there is some $y \in U_{k-1}^{\prime} \cap S x$ such that $y \notin U_{k-2}^{\prime}$. (Here and below we let $U_{0}^{\prime}=\varnothing$.) This is so because $x \notin U_{k-1}^{\prime}$ implies that there is an element $y \in S x$ such that $S y \varsubsetneqq S x$ and such that $y \notin U_{k-2}^{\prime}$. Choosing $y$ so that $S y$ is minimal with respect to this property implies that $y \in U_{k-1}^{\prime}$.

Let $a_{n} \in U_{n}^{\prime}$ such that $a_{n} \notin U_{n-1}^{\prime}$. Using the above observation inductively, one finds $a_{i} \in U_{i}^{\prime}$ for $i=1, \cdots, n$, such that $a_{i} \notin U_{i-1}^{\prime}$ and such that $S a_{1} \subsetneq S a_{2} \varsubsetneqq \cdots \subsetneq S a_{n}$.

Let us now consider the left $S$-set $U_{n}=U_{n}^{\prime} / U_{1}^{\prime}$. Denote the image of $U_{i}^{\prime}$ in $U_{n}$ under the natural homomorphism by $U_{i}$ for $i=1, \cdots, n$, and denote the class of $U_{1}^{\prime}$ by 0 . Hence we have a proper chain of $S$-subsets of $U_{n}$ given by $\{0\}=U_{1} \varsubsetneqq U_{2} \subsetneq \cdots \sqsubseteq U_{n}$. We may 
continue to write nonzero elements of $U_{n}$ the same as elements of $U_{n}^{\prime}$; thus we write $a_{i} \in U_{i}$ for $i=2, \cdots, n$.

Finally we form the left $S$-set $U_{n} \times\left\{1, \cdots, 2^{n-1}\right\}$ by letting $s(x, \alpha)=(s x, \alpha)$ for $s \in S, x \in U_{n}$ and $1 \leqq \alpha \leqq 2^{n-1}$, and then we set $V_{n}=\left(U_{n} \times\left\{1, \cdots, 2^{n-1}\right\}\right) / \sim$ where $\sim$ is the congruence relation given by $(x, \alpha) \sim(y, \beta)$ if and only if $x=y \in U^{k}$ for some $k$ and $2^{n-k} p<$ $\alpha, \beta \leqq 2^{n-k}(p+1)$ for some $p$ with $0 \leqq p<2^{k-1}$. (One wishes to use the smallest possible $k$ here.)

Observe that this implies that if $x \in U_{n}$ and $x \notin U_{n-1}$, then $(x, \alpha) \sim(x, \beta)$ only when $\alpha=\beta$, while for $x=0$, i.e., $x \in U_{1},(x, \alpha) \sim$ $(x, \beta)$ for all $1 \leqq \alpha, \beta \leqq 2^{n-1}$. We can picture $V_{n}$ as a binary tree such that every path from a leaf to the root looks like a copy of $U_{n}$. See Figure 2. Denote the class of $(x, \alpha)$ in $V_{n}$ by $[x, \alpha]$. We have a natural epimorphism of $S$-sets $\pi: V_{n} \rightarrow U_{n}$ given by $\pi([x, \alpha])=x$.

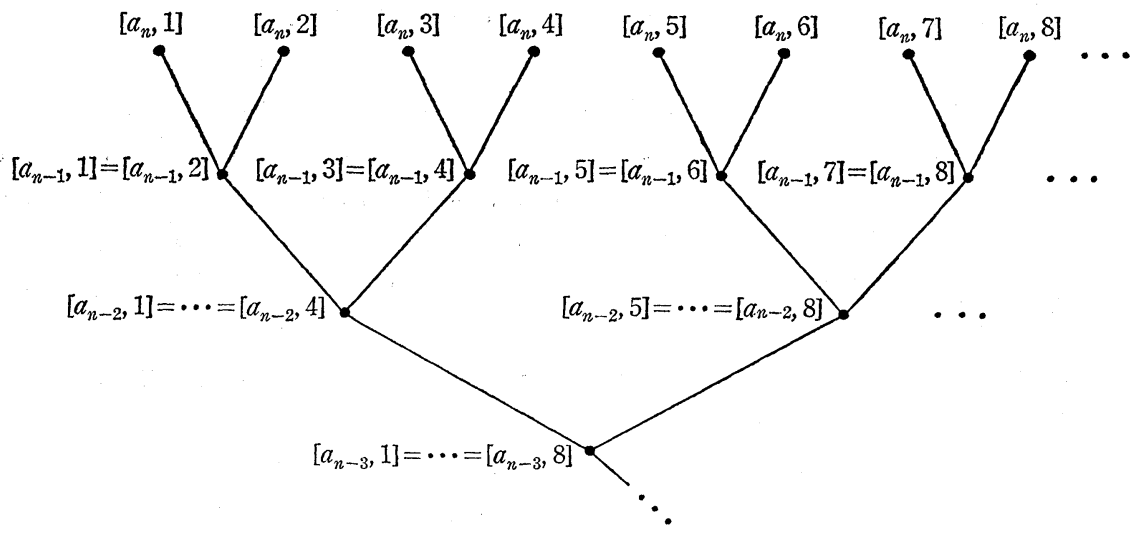

FIGURE 2

Let us now record properties of $V_{n}$ in the following lemmas. By convention $U_{0}=\varnothing$.

LEMMA 2.1.1. Let $[x, \alpha] \in \pi^{-1}\left(U_{k}\right)$ with $[x, \alpha] \notin \pi^{-1}\left(U_{k-1}\right)$ for some $1 \leqq k \leqq n$.

(i) For every $[y, \beta] \in V_{n}$ either $[x, \alpha] \in S[y, \beta]$ or $S[x, \alpha] \cap$ $S[y, \beta] \subset \pi^{-1}\left(U_{k-1}\right)$. Thus $[x, \alpha]$ is $\pi^{-1}\left(U_{k-1}\right)$-distinguished.

(ii) $[y, \beta] \in \pi^{-1}\left(U_{k}\right)$ with $[y, \beta] \notin \pi^{-1}\left(U_{k-1}\right)$, then either $S[x, \alpha]=$ $S[y, \beta]$ or $[x, \alpha]$ and $[y, \beta]$ are separated in $\pi^{-1}\left(U_{k}\right)$ and $S[x, \alpha] \cap$ $S[y, \beta] \subset \pi^{-1}\left(U_{k-1}\right)$.

Proof. If $[z, \gamma] \in S[x, \alpha] \cap S[y, \beta]$, then by construction of $V_{n}$, $[z, \gamma]=[z, \alpha]=[z, \beta]$ and $z \in S x \cap S y$ in $U_{n}$. But $x \in U_{k}$ implies that $z \in U_{k}$. If $z \notin U_{k-1}$, then by construction of $U_{k}, S x=S z$. Thus 
$[x, \alpha] \in S[x, \alpha]=S[z, \alpha] \subset S[y, \beta]$. Hence if $[x, \alpha] \notin S[y, \beta]$, then $z \in U_{k-1}$, which says $S[x, \alpha] \cap S[y, \beta] \subset \pi^{-1}\left(U_{k-1}\right)$. This gives (i).

For (ii) suppose $[x, \alpha],[y, \beta] \in S[w, \delta]$ for some $[w, \delta] \in \pi^{-1}\left(U_{k}\right)$. Then as in proof of (i) we show $S[x, \alpha]=S[y, \beta]=S[w, \delta]$. Hence if $[x, \alpha]$ and $[y, \beta]$ are not separated in $\pi^{-1}\left(U_{k}\right)$, then $S[x, \alpha]=S[y, \beta]$. This gives (ii).

Lemma 2.1.2. (i ) For $1 \leqq k \leqq n-1,\left\{\left[a_{n-k}, 2^{k} p+1\right]: 0 \leqq p<\right.$ $\left.2^{n-k-1}\right\} \subset\left(V_{n}\right)_{k} \subset \pi^{-1}\left(U_{n-k}\right)$.

$$
\text { (ii) } \operatorname{sl}\left(V_{n}\right)=n \text {. }
$$

Proof. (i) implies (ii), since (i) for $k=n-1$ implies that $\phi \neq\left(V_{n}\right)_{n-1} \subset \pi^{-1}\left(U_{1}\right)=\{0\}$ which implies $\left(V_{n}\right)_{n}=\varnothing$. Thus $\operatorname{sl}\left(V_{n}\right)=n$.

We establish (i) by induction on $k$. For $k=1$, we see by Lemma 2.1.1 that the set $\left\{[x, \alpha]:[x, \alpha] \in \pi^{-1}\left(U_{n}\right)=V_{n}\right.$ but $[x, \alpha] \notin$ $\left.\pi^{-1}\left(U_{n-1}\right)\right\}$ generates maximal orbits in $V_{n}$ and that the intersection of orbits generated by a separated pair of elements is contained in $\pi^{-1}\left(U_{n-1}\right)$. Hence $\left(V_{n}\right)_{1} \subset \pi^{-1}\left(U_{n-1}\right)$.

On the other hand, $\left[a_{n}, 2 p+1\right]$ and $\left[a_{n}, 2(p+1)\right]$ are separated in $V_{n}$ for $0 \leqq p<2^{n-2}$ by Lemma 2.1.1. Thus $\left[a_{n-1}, 2 p+1\right] \epsilon$ $S\left[a_{n}, 2 p+1\right] \cap S\left[a_{n}, 2(p+1)\right] \subset\left(V_{n}\right)_{1}$. This gives (i) for the case $k=1$.

Suppose by induction that (i) is true for $1<k<n-1$. We establish it for $k+1$.

Since $\left(V_{n}\right)_{k} \subset \pi^{-1}\left(U_{n-k}\right)$, we see that if $[x, \alpha]$ and $[\mathrm{y}, \beta] \in\left(V_{n}\right)_{k}$ are separated in $\pi^{-1}\left(U_{n-k}\right)$, then they are separated in $\left(V_{k}\right)_{k}$ and by Lemma 2.1.1 $S[x, \alpha] \cap S[y, \beta] \subset \pi^{-1}\left(U_{n-k-1}\right)$. Thus $\left(V_{n}\right)_{k+1} \subset$ $\pi^{-1}\left(U_{n-(k+1)}\right)$.

Finally, if $\left\{\left[a_{n-k}, 2^{k} p+1\right]: 0 \leqq p<2^{n-k-1}\right\} \subset\left(V_{n}\right)_{k}$, we observe by Lemma 2.1.1 that $\left[a_{n-k}, 2^{k}(2 p)+1\right]$ and $\left[a_{n-k}, 2^{k}(2 p+1)+1\right]$ are separated in $\left(V_{n}\right)_{k}$ for $0 \leqq p<2^{n-k-2}$. Thus $\left[a_{n-k-1}, 2^{k+1} p+1\right] \epsilon$ $S\left[a_{n-k}, 2^{k}(2 p)+1\right] \cap S\left[a_{n-k}, 2^{k}(2 p+1)+1\right] \subset\left(V_{n}\right)_{k+1}$ for $0 \leqq p<2^{n-k-2}$. This completes the lemma.

Lemma 2.1.3. (i ) For $1 \leqq k \leqq n-1, \quad\left(V_{n}\right)^{k} \supset \pi^{-1}\left(U_{k}\right) \quad$ but $\left[a_{k+1}, 1\right] \notin\left(V_{n}\right)^{k}$.

$$
\text { (ii) } h\left(V_{n}\right)=n \text {. }
$$

Proof. To see that (ii) follows from (i) we see that when $k=$ $n-1$ we have $V_{n} \supsetneq\left(V_{n}\right)^{n-1} \supset \pi^{-1}\left(U_{n-1}\right)$. But by Lemma 2.1.1 every remaining element in $V_{n}$ will be $\pi^{-1}\left(U_{n-1}\right)$-distinguished and hence $\left(\left(V_{n}\right)^{n-1}\right)$-distinguished. This gives $\left(V_{n}\right)^{n}=V_{n}$, which says $h\left(V_{n}\right)=n$.

We establish (i) by induction on $k$. For $k=1, \pi^{-1}\left(U_{1}\right)=\{0\}$, a 
fixed point, so that $\left(V_{n}\right)^{1} \supset\{0\}=\pi^{-1}\left(U_{1}\right)$. However, by Lemma 2.1.1, $\left[a_{2}, 1\right]$ and $\left[a_{2}, 2^{n-2}+1\right]$ generate orbits which are incomparable under inclusion. Yet $0=\left[a_{1}, 1\right] \in S\left[a_{2}, 1\right] \cap S\left[a_{2}, 2^{n-2}+1\right]$. This assures us that $\left[a_{2}, 1\right] \notin\left(V_{n}\right)^{1}$.

Suppose by induction that (i) is true for $1<k<n-1$. Thus $\left(V_{n}\right)^{k} \supset \pi^{-1}\left(U_{k}\right)$. By Lemma 2.1.1 every element of $\pi^{-1}\left(U_{k+1}\right)$ is either in $\pi^{-1}\left(U_{k}\right)$ or is $\pi^{-1}\left(U_{k}\right)$-distinguished. Hence if $[x, \alpha] \notin\left(V_{n}\right)^{k}$ but $[x, \alpha] \in \pi^{-1}\left(U_{k+1}\right)$, then $S[x, \alpha]$ is a $\pi^{-1}\left(U_{k}\right)$-distinguished orbit, and hence a $\left(V_{n}\right)^{k}$-distinguished orbit. Thus $[x, \alpha] \in\left(V_{n}\right)^{k+1}$, which gives $\pi^{-1}\left(U_{k+1}\right) \subset\left(V_{n}\right)^{k+1}$.

On the other hand, if $\left[a_{k-1}, 1\right] \notin\left(V_{n}\right)^{k}$, then since $\left[a_{k+1}, 1\right] \in$ $S\left[a_{k+2}, 1\right] \cap S\left[a_{k+2}, 2^{n-k-2}+1\right]$ and since $S\left[a_{k+2}, 1\right]$ and $S\left[a_{k+2}, 2^{n-k-2}+1\right]$ are incomparable under inclusion, we see that $\left[a_{k+2}, 1\right] \notin\left(V_{n}\right)^{k+1}$. This concludes the induction.

Finally, to complete the proof that (i) implies (ii) in Theorem 2.1 , let us suppose that $S$ contains chains of principal left ideals of all lengths. Thus for each $n \geqq 1$ we can construct an $S$-set $V_{n}$ as above. Notice that each $V_{n}$ has a fixed point $0_{n} \in V_{n}$. Let $X$ be the 0 -disjoint union of the $\left\{V_{n}: n \geqq 1\right\}$, that is, $X=\left(\bigcup_{n=1}^{\infty} V_{n}\right) / \approx$ where $\bigcup_{n=1}^{\infty} V_{n}$ is the disjoint union of the $V_{n}$ and where $\approx$ is the congruence on this disjoint union given by $v \approx v^{\prime}$ if and only if $v=v^{\prime}$ or $v=0_{n}$ and $v^{\prime}=0_{m}$ for some $m$ and $n$.

To compute $h(X)$ one finds that $X^{1}=\{0\}$ and that $X^{k}=$ $\left(\bigcup_{n=1}^{\infty}\left(V_{n}\right)^{k}\right) / \approx$. Thus for every integer $k$ one has $X^{k} \neq X$, but $\bigcup_{k=1}^{\infty} X^{k}=X$. This gives $h(X)=\omega$.

However one also sees for every integer $k$ that $\{0\} \in X_{k}=$ $\left(\bigcup_{n=1}^{\infty}\left(V_{n}\right)_{k}\right) / \approx$. Hence $\{0\}=\bigcap_{k=1}^{\infty} X_{k}=X_{\omega}$. But then $X_{\omega+1}=\varnothing$, which says that $\operatorname{sl}(X)=\omega+1>h(X)$ contrary to (i). This completes the proof of Theorem 2.1.

REMARK. To see that the result fails if one simply demands that $S$ satisfy both the ascending and descending chain condition on principal left ideals, or even both chain conditions on orbits, or if in ( $v$ ) one only demands that $d(a)<\infty$ for all $a \in S$, we can consider the following example. Let $C_{n}=\left\{a_{n}, a_{n}^{2}, \cdots, a_{n}^{n-1}, a_{n}^{n}=0_{n}\right\}$ be finite, cyclic, nil semigroups. Let $S=\left(\bigcup_{n=1}^{\infty} C_{n}\right) \cup\{1\}$ where 1 is a two-sided identity and where we set $a_{n}^{k} a_{m}^{l}=0_{m}$ for $m \neq n$ and multiply as in $C_{n}$ otherwise. Then $S$ satisfies both chain conditions on principal left ideals and on orbits and every $a \in S$ is such that $d(a)<\infty$. But $h(1)=\infty$ and $S$ chains of principal left ideals of arbitrary length.

3. Monoids bounded by $n$. In order to analyze in more detail what can happen let us call a monoid $S$ bounded by $n \geqq 0$ if for every $S$-set $X$ we have $\operatorname{sl}(X) \leqq h(X) \leqq s l(X)+n$. In [3] it was 
shown that for every given positive integer $n$ there is a monoid $S$ and an $S$-set $X$ such that $h(X)=s l(X)+n$.

Lemma 3.1. If $S$ is a bounded monoid and if $S$ contains a proper chain of principal left ideals of length $n+2$ for an integer $n \geqq 0$, then there exists a left $S$-set such that $h(X)=\operatorname{sl}(X)+n$.

Proof. Let us note first that the condition of the lemma is equivalent to the statement that $h(1) \geqq n+2$ in $S$.

Since $S$ is bounded, it satisfies both chain conditions on principal left ideals. We can use a construction similar to that used in Proposition 1.1 to create an $S$-set $X$ such that $s l(X)=2$ and $h(X)=$ $n+2$. Suppose that we have $S a_{0} \supsetneq S a_{1} \supsetneq \cdots \supsetneq S a_{n+1}$ a proper chain of principal left ideals. Since $S$ is bounded, we may assume that the $a_{i}$ are chosen so that $h\left(a_{0}\right)=n+2$ in $S$. This guarantees that $S a_{0}$ contains no longer proper chain of principal left ideals, that $S a_{n+1}$ is minimal, and that each $S a_{i}$ is minimal among principal left ideals properly containing $S a_{i+1}$ for $i=0, \cdots, n$. Now as in Proposition 1.1 we set $X=\left(S a_{0} \times\{0, \cdots, n+1\}\right) / \sim$ where $(x, \alpha) \sim$ $(x, \beta)$ if $x \in S a_{i}$ and $\alpha, \beta \leqq i$ for $0 \leqq i \leqq n+1$. We denote the class of $(x, \alpha)$ in $X$ by $[x, \alpha]$.

In $X$ the set $\left\{\left[a_{0}, \alpha\right]: 0 \leqq \alpha \leqq n-1\right\}$ is a set of generators for all the distinct maximal orbits of $X$. As in Proposition 1.1 we see that $X_{1}=S\left[a_{1}, 0\right]$ since $[x, \gamma] \in S\left[a_{0}, \alpha\right] \cap S\left[a_{0}, \beta\right]$ for $\alpha \neq \beta$ implies that $[x, \gamma]=[x, 0]$ and $x \in S a_{1}$, and since $\left[a_{1}, 0\right] \in S\left[a_{0}, 0\right] \cap S\left[a_{0}, 1\right]$. Thus since $X_{1}$ is an orbit, $X_{2}=\varnothing$, which gives $s l(X)=2$.

In order to show that $h(X)=n+2$, we can first observe that if we define a function $h_{X}: X \rightarrow N$ as in the proof that (iv) implies (iii) in Theorem 2.1, then $h_{X}\left(\left[\alpha_{0}, \alpha\right]\right)=h_{S}\left(a_{0}\right)=n+2$. Thus as in the proof of Theorem 2.1, we can conclude that $h(X) \leqq n+2$. It now suffices to show that for $k<n+2$, we have $\left[a_{n-1-k}, 0\right] \notin X^{k}$. This we do by induction on $k$. For $k=0$, there is nothing to prove since $X^{0}=\varnothing$. Thus suppose that for $0 \leqq k<n+1$ we have $\left[a_{n-1-k}, 0\right] \notin X^{k}$. By construction of $X$ we see that $\left[a_{n-1-k}, 0\right]=$ $\left[a_{n-1-k}, n-1-k\right] \in S\left[a_{0}, n-1-k\right]$. However $\left[a_{n-1-k}, 0\right] \in S\left[a_{n-2-k}, 0\right]$, and $X, S\left[a_{n-2-k}, 0\right]$ and $S\left[a_{0}, n-1-k\right]$ are incomparable under inclusion. Thus since $\left[a_{n-1-k}, 0\right] \in S\left[a_{n-2-k}, 0\right] \cap S\left[a_{0}, n-1-k\right]$ and since $\left[a_{n-1-k}, 0\right] \notin X^{k}$, we find that $\left[a_{n-1-(k+1)}, 0\right] \notin X^{k+1}$, which completes the induction. Hence letting $k=n+1$ we get $\left[a_{0}, 0\right] \notin X^{n+1}$ while $X=X^{n+2}$, so that $h(X)=n+2$ as desired.

We can now characterize monoids bounded by $n$.

THEOREM 3.2. Let $n \geqq 0$ be an integer. A monoid $S$ is bounded by $n$ if and only if $S$ contains no proper chain of principal left 
ideals of length exceeding $n+2$. Furthermore, if there is a chain of this length, then there is a left $S$-set $X$ such that $h(X)=$ $\operatorname{sl}(X)+n$.

Proof. By Theorem 2.1 we know that $\operatorname{sl}(X) \leqq h(X)$ for every left $S$-set $X$ if and only if the lengths of chains of principal left ideals in $S$ are bounded. If $S$ contains a chain of principal left ideals of length $n+2$, then Lemma 3.1 produces a left $S$-set $X$ such that $\operatorname{sl}(X) \leqq h(X)=s l(X)+n$. Thus the inequality $h(X) \leqq$ $s l(X)+n$ for every $S$-set $X$ implies that $S$ contains no proper chain of principal left ideals of length exceeding $n+2$.

It remains to show that if every chain of principal left ideals in $S$ is of length not exceeding $n+2$, then for every left $S$-set $X$ we have $h(X) \leqq s l(X)+n$. Now as in Lemma 3.1 we observe that our condition says that $h(1) \leqq n+2$ in $S$ and as in the proof of Lemma 3.1 and Theorem 2.1, this implies that $h(X) \leqq n+2$ for every left $S$-set $X$. Since chains of principal left ideals are bounded in $S$, one finds that $S$ satisfies both chain conditions on orbits. Thus $s l(X)=0$ if and only if $X=\varnothing$, which is if and only if $h(X)=0$. Hence we need only consider the case $s l(X)=1$. But $s l(X)=1$ implies that $X$ is a disjoint union of orbits. Thus it suffices to suppose that $X$ is a single orbit, say, $X=S x_{0}$.

As in Lemma 3.1 and the proof that (iv) implies (iii) in Theorem 2.1 we can define $h_{X}: X \rightarrow N$ and find that $h_{X}\left(x_{0}\right) \leqq h_{S}(1) \leqq n+2$ and that $X_{k} \supset\left\{x \in X: h_{X}(x) \leqq k\right\}$ for $k>0$. Thus $X^{n} \supset\left\{x \in X: h_{X}(x) \leqq n\right\}$. Suppose now that $x \notin X^{n}$. Then $h_{X}(x) \geqq n+1$. If $h_{X}(x)=n+2$, then since $h_{X}(x) \leqq h_{X}\left(x_{0}\right) \leqq n+2$, we must have $S x=S x_{0}=X$. Thus $y \in S x$ for all $y \notin X^{n}$, and $x$ is $X^{n}$-distinguished. If $h_{X}(x)=n+1$ and if $y \notin X^{n}$ is such that $x \notin S y$, then we must have $h_{X}(y)=n+1$. Thus either $y \in S x$ or $h_{x}(z) \leqq n$ for all $z \in S x \cap S y$ since $S x \cap S y \varsubsetneqq S x$ and $S x \cap S y \varsubsetneqq S_{y}$. But this says $S x \cap S y \subset X^{n}$. Hence every element of $X$ is $X^{n}$-distinguished. Therefore $X=S x_{0}$ is an $X^{n}$-distinguished orbit so that $X=X^{n+1}$. This shows that $h(X) \leqq n+1=n+\operatorname{sl}(X)$ as desired.

4. Further properties. Let $S$ be a bounded monoid and let $h(1)=n$, where $h$ is the height function on elements of $S$. Let $I_{k}=\{x \in S: h(x) \leqq k\}, 1 \leqq k \leqq n$. Then we can observe the following properties of $S$.

Proposition 4.1. Let $S, h, n$, and $I_{k}$ be as above.

(i) For all $x, y \in S$, one has $h(x y) \leqq h(x)$ and $h(x y) \leqq h(y)$.

(ii) Each $I_{k}$ is a two-sided ideal in $S$.

(iii) $I_{n-1}$ consists of the nonunits of $S$. 
(iv) $x \in S$ is a unit if and only if $h(s)=h(1)=n$.

(v) If $x \mathscr{J} y$ in $S$, then $h(x)=h(y)$.

Proof. (i) Since $x y \in S y$, it is clear that $h(x y) \leqq h(y)$. On the other hand, since one has an $S$-map $\phi: S x \rightarrow S x y$ given by $\phi(t)=t y$ for $t \in S x$, the inverse image of any proper chain of principal left ideals contained in $S x y$ gives rise to such a chain contained in $S x$. Hence $h(x y) \leqq h(x)$.

(ii) By (i) if $h(x) \leqq k$, then $h(a x), h(x a) \leqq h(x) \leqq k$ for all $a \in S$. Thus each $I_{k}$ is a two-sided ideal.

(iii) and (iv). For $x \in S, h(x)=h(1)=n$ if and only if $S x=S$, i.e., if and only if $x$ has a left inverse. But if $a \in S$ is a left inverse of $x$, then $a x=1$ and by (i) $n=h(1)=h(a x) \leqq h(a) \leqq n$, so that $a$ also has a left inverse. Thus $x a=1$ and both are two-sided units.

(v) If $x \mathscr{J} y$ in $S$, then $S x S=S y S$ so that $x \in I_{k}$ if and only if $y \in I_{k}$ for $1 \leqq k \leqq n$. But since $x \in I_{k}$ for $k=h(x)$ and $y \in I_{k}$ for $k=h(y)$ we get $h(x)=h(y)$ as desired.

We should observe that $S$ being bounded is not a strong enough condition for $S$ to satisfy $\mathscr{J}=\mathscr{D}$. If $S=T \cup\{1\}$ where 1 is an identity and $T$ is a simple, idempotent free semigroup which contains a minimal left ideal, then $S$ is bounded (in fact, $h(1)=2$ ), but $\mathscr{J} \neq \mathscr{D}$ (see [1], v. 2, Ex. 1, p. 93).

Suppose now that $S$ is a finite monoid. Then certainly $S$ is bounded, and the bounds expressed in Theorem 3.2 apply to any $S$-set. Suppose further that we consider $S$ as a left $S$-set over itself. Then since $S$ is a single orbit, we always have $\operatorname{sl}(S)=1$. However, the height of this $S$-set need not be 1 , and in fact the difference $h(S)-s l(S)$ gives some measure of the complexity of the left ideal structure of $S$. If $S$ is cyclic, then $h(S)=\operatorname{sl}(S)=1$. However, we show in conclusion that for some $S$, the difference $h(S)-s l(S)$ attains the limit imposed by Theorem 3.2 .

Proposition 4.2. Let $n \geqq 0$ and let $T_{n+2}$ be the monoid of all transformation on the set $\{1, \cdots, n+2\}$ (with functions written on the left). Then letting $T_{n+2}$ act on itself from the left we see that $\operatorname{sl}\left(T_{n+2}\right)=1$ and $h\left(T_{n+2}\right)=n+1$.

Proof. That $s l\left(T_{n+2}\right)=1$ follows since $T_{n+2}$ is a single orbit over itself. Also since $T_{n+2}$ satisfies the hypotheses of Theorem 3.2 and is an orbit over itself, we may conclude as a corollary to the proof that $h\left(T_{n+2}\right) \leqq n+1$. Thus it suffices to show that $\left(T_{n+2}\right)^{n} \neq T_{n+2}$.

Let $\alpha_{1}, \cdots, \alpha_{n+1}, \beta_{2}, \cdots, \beta_{n+1} \in T_{n+2}$ be given by 


$$
\alpha_{k}(x)=\left\{\begin{array}{ll}
x & 1 \leqq x \leqq k \\
k & k<x
\end{array} \text { and } \quad \beta_{k}(x)= \begin{cases}x & 1 \leqq x \leqq k-1 \\
k-1 & x=k \\
k & k<x\end{cases}\right.
$$

for $1 \leqq x \leqq n+2$. One easily verifies that $\alpha_{k} \alpha_{k+1}=\alpha_{k}$ and that $\alpha_{k} \beta_{k+1}=\alpha_{k}$ for $1 \leqq k \leqq n$. Furthermore, one sees that all the principal left ideals $T_{n+2} \alpha_{\kappa}, 1 \leqq k \leqq n+1$, and $T_{n+2} \beta_{k}, 2 \leqq k \leqq n+1$ are distinct since two elements of $T_{n+2}$ generate the same principal left ideal if and only if they induce the same partition on $\{1, \cdots, n+2\}$. (See [1], v. 1, §2.2.) Thus one has $T_{n+2} \alpha_{n+1} \supsetneq \cdots \supsetneq T_{n+2} \alpha_{2} \supsetneq T_{n+2} \alpha_{1}$, $T_{n+2} \beta_{k+1} \supsetneq T_{n+2} \alpha_{k}$ for $k=1, \cdots, n$, and $T_{n+2} \beta_{k+1}$ and $T_{n+2} \alpha_{k+1}$ are incomparable under inclusion for $k=1, \cdots, n$. Thus since $\alpha_{k} \in$ $T_{n+2} \alpha_{k+1} \cap T_{n+2} \beta_{k+1}$ for $k=1, \cdots, n$, we can conclude by induction that $\alpha_{k} \notin\left(T_{n+2}\right)^{k-1}$ for $k=1, \cdots, n$. Hence $\alpha_{n+1} \notin\left(T_{n+2}\right)^{n}$ so that $\left(T_{n+2}\right)^{n} \neq\left(T_{n+2}\right)$. Therefore $h\left(T_{n+2}\right)=n+1$ as desired.

\section{REFERENCES}

1. A. H. Clifford and G. B. Preston, The Algebraic Theory of Semigroups, vol. 1 and Amer. Math. Soc., Providence, 1961 and 1967.

2. W. R. Nico, A study of operands in terms of maximal generalized orbits, J. Algebra, 30 (1974), 473-484.

3. - Height of operands over monoids satisfying the d.c.c. on orbits, Proc. Amer. Math. Soc., 48 (1975), 313-320.

Received November 23, 1976 and in revised form September 2, 1977.

TUlane UNIVERSiTy

New ORLeans, LA 70118 


\section{PACIFIC JOURNAL OF MATHEMATICS}

\section{EDITORS}

RICHARD ARENS (Managing Editor)

University of California

Los Angeles, CA 90024

Charles W. Curtis

University of Oregon

Eugene, OR 97403

C. C. Moore

University of California

Berkeley, CA 94720

\section{J. DugundJI}

Department of Mathematics

University of Southern California

Los Angeles, CA 90007

R. FInN and J. Milgram

Stanford University

Stanford, CA 94305

\section{ASSOCIATE EDITORS}
E. F. BECKENBACH
B. H. NeumanN
F. WOLF
K. YoSHIDA

\section{SUPPORTING INSTITUTIONS}

\author{
UNIVERSITY OF BRITISH COLUMBIA \\ CALIFORNIA INSTITUTE OF TECHNOLOGY \\ UNIVERSITY OF CALIFORNIA \\ MONTANA STATE UNIVERSITY \\ UNIVERSITY OF NEVADA, RENO \\ NEW MEXICO STATE UNIVERSITY \\ OREGON STATE UNIVERSITY \\ UNIVERSITY OF OREGON
}

\author{
UNIVERSITY OF SOUTHERN CALIFORNIA \\ STANFORD UNIVERSITY \\ UNIVERSITY OF HAWAII \\ UNIVERSITY OF TOKYO \\ UNIVERSITY OF UTAH \\ WASHINGTON STATE UNIVERSITY \\ UNIVERSITY OF WASHINGTON
}

The Supporting Institutions listed above contribute to the cost of publication of this Journal, but they are not owners or publishers and have no responsibility for its content or policies.

Mathematical papers intended for publication in the Pacific Journal of Mathematics should be in typed form or offset-reproduced, (not dittoed), double spaced with large margins. Please do not use built up fractions in the text of the manuscript. However, you may use them in the displayed equations. Underline Greek letters in red, German in green, and script in blue. The first paragraph or two must be capable of being used separately as a synopsis of the entire paper. Items of the bibliography should not be cited there unless absolutely necessary, in which case they must be identified by author and journal, rather than by item number. Manuscripts, in triplicate, may be sent to any one of the editors. Please classify according to the scheme of Math. Reviews, Index to Vol. 39. All other communications should be addressed to the managing editor, or Elaine Barth, University of California, Los Angeles, California, 90024.

50 reprints to each author are provided free for each article, only if page charges have been substantially paid. Additional copies may be obtained at cost in multiples of 50 .

The Pacific Journal of Mathematics is issued monthly as of January 1966. Regular subscription rate: $\$ 72.00$ a year (6 Vols., 12 issues). Special rate: $\$ 36.00$ a year to individual members of supporting institutions.

Subscriptions, orders for numbers issued in the last three calendar years, and changes of address should be sent to Pacific Journal of Mathematics, 103 Highland Boulevard, Berkeley, California, 94708. Older back numbers obtainable from Kraus Periodicals Co., Route 100, Millwood, NY 10546.

PUBLISHED BY PACIFIC JOURNAL OF MATHEMATICS, A NON-PROFIT CORPORATION

Printed at Kokusai Bunken Insatsusha (International Academic Printing Co., Ltd.). 8-8, 3-chome, Takadanobaba, Shinjuku-ku, Tokyo 160, Japan.

Copyright (C) 1978 by Pacific Journal of Mathematics

Manufactured and first issued in Japan 


\section{Pacific Journal of Mathematics

Vol. 74, No. $2 \quad$ June, 1978

Aharon Atzmon, Spectral synthesis in some spaces of bounded continuous

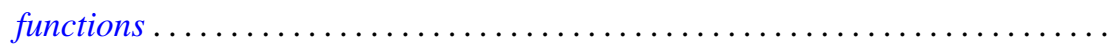

Karl Egil Aubert and Isidor Fleischer, Tensor products of ideal systems and their modules.............................................

Richard F. Basener, Several dimensional properties of the spectrum of a uniform

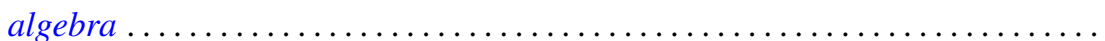

R. H. Bing and Michael Peter Starbird, Super triangulations ............. 307

Andrew Carson, Coherent polynomial rings over regular rings of finite index .....

Robert M. DeVos and Frederick W. Hartmann, Sequences of bounded summability domains .................................................

George Grätzer and R. Padmanabhan, Symmetric difference in abelian groups ....

Robert L. Griess, Jr., A remark about groups of characteristic 2-type and

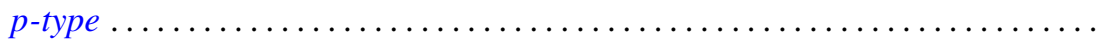

Emil Grosswald and F. J. Schnitzer, A class of modified $\zeta$ and L-functions........

Jutta Hausen and Johnny Albert Johnson, Ideals and radicals of some

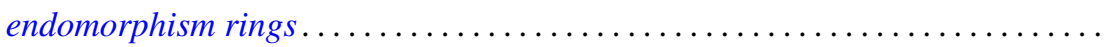

Jean Ann Larson, A solution for scattered order types of a problem of Hagendorf ............................................

Peter A. McCoy, Extremal properties of real biaxially symmetric potentials in $E^{2(\alpha+\beta+2)}$

Héctor Alfredo Merklen, Hereditary crossed product orders .

Hal G. Moore and Adil Mohamed Yaqub, Equational definability of addition in certain rings...

Robert Laurens Moore, Reductivity in $C^{*}$-algebras and essentially reductive operators. . .

Joseph Alvin Neisendorfer, Lie algebras, coalgebras and rational homotopy theory for nilpotent spaces...

William Raymond Nico, Bounded monoids

Richard Paul Osborne, Simplifying spines of 3-manifolds ...

Richard Paul Osborne, The simplest closed 3-manifolds. With an appendix by Osborne and J. Yelle.

Clayton Collier Sherman, The $K$-theory of an equicharacteristic discrete valuation ring injects into the $K$-theory of its field of quotients.... .

Mitchell Herbert Taibleson, The failure of even conjugate characterizations of $H^{1}$

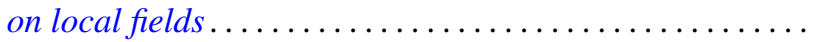

Keti Tenenblat, On characteristic hypersurfaces of submanifolds in Euclidean space ...................................

Jeffrey L. Tollefson, Involutions of Seifert fiber spaces..........

Joel Larry Weiner, An inequality involving the length, curvature, and torsions of a curve in Euclidean $n$-space .......................

Neyamat Zaheer, On generalized polars of the product of abstract homogeneous polynomials.... 